\title{
BUCKLING OF COMPOSITE NONLOCAL OR GRADIENT CONNECTED BEAMS
}

\author{
NOËL CHALLAMEL ${ }^{*}$, , ISMAIL MECHAB ${ }^{\dagger, \S}$, \\ NOUREDDINE EL MEICHE ${ }^{\dagger, \pi}$ and BAGHDAD KROUR ${ }^{\dagger, \|}$ \\ *Université Européenne de Bretagne, INSA de Rennes-LGCGM \\ 20, Avenue des Buttes de Coësmes, \\ 35708 Rennes Cedex 7 - France \\ ${ }^{\dagger}$ Department of Civil Engineering, Université de Sidi Bel Abbes \\ Laboratoire des Matériaux et Hydrologie-Algerie \\ "noel.challamel@insa-rennes.fr \\ \$ismyala@yahoo.fr \\ "nouryel@hotmail.com \\ "Kr_bag@yahoo.fr
}

Received 20 July 2010

Accepted 19 August 2010

\begin{abstract}
The buckling of an axially loaded elastic composite beam with a nonlocal core or a nonlocal connection system is studied in this paper. The composite beam or the sandwich beam is composed of two Euler-Bernoulli beams with a nonlocal elastic interaction. This nonlocal interaction is physically based on the Reissner's model based on three-parameters' interaction function. The energy equations are first presented, and the differential equations are rigorously obtained from a variational principle. We show that the connection model can be expressed in an integral format, therefore, inducing the nonlocal character of this beam elastic interaction model. Furthermore, the variational format of this nonlocal composite model is given, leading to meaningful natural and higher-order boundary conditions. The system of these differential equations can be reduced to a single 10th-order differential equation. We present an exact method to solve this stability problem, based on Ferrari or Cardano's method. The solution can be fully simplified in case of specific boundary conditions with symmetrical considerations. The stability domain is analytically characterized in the loading space for the pinned-pinned boundary conditions. The correspondence between the buckling of the nonlocal composite column and the shear composite column is discussed. Finally, it is shown that the Timoshenko beam model is a nonlocal integral model.
\end{abstract}

Keywords: Sandwich beams; nonlocal mechanics; Reissner's model; buckling; higher-order boundary conditions; gradient elasticity model; composite beams.

\section{Introduction}

Sandwich beams or composite connected beams are composed of two interacting beams. There are many applications of such tri-layer composite structural members 
in civil engineering, but also in aerospace or mechanical engineering. Layered structural elements with interlayer slip are typically encountered in wood design, where wooden beams are made up of layers assembled by means of nailing, bolting, or gluing (with a soft shear modulus). Partially composite structures built up by subelements of different materials and connected by shear connectors to form an interacting unit, such as timber-concrete or steel-concrete elements, are widely used in building engineering. In the case of a flexible connection, the analysis procedure requires consideration of the interlayer slip between the subelements, leading to the partial interaction concept.

The analysis of these composite structural members can be mainly gathered into two categories. In the first category, the composite beam can be modeled by an equivalent shear beam with first-order or higher-order theories (this is for instance the approaches of Haringx, Engesser - see Refs. 1-3, or the higher-order theories of Reddy - see for instance Ref. 4). In the second category, the composite beam is subdivided into beams or shear layers with some possible kinematic discontinuities at the boundary of each layer. Sandwich beam can be modeled with such a tri-layer model. Sandwich beams are usually composed of three layers, two thin faces and a thick weak shear core ${ }^{5-8}$ see also Refs. 9 and 10. The buckling solution of such a sandwich column has been obtained by Hoff and Mautner. ${ }^{11}$ In this last family of structural modeling, improved complexity is generally located on the beam kinematics (with Timoshenko beam elements for each beam), shear layer constitutive behavior with some possible inelastic phenomena (damage or plasticity phenomena), or the possible flexible core. ${ }^{12,13}$ The constitutive behavior of the core is mainly reduced to a local elastic behavior with some simplified kinematics. However, the core plays a crucial role in the global stability behavior, and a high-order theory is needed to improve the accuracy of the solution.

In this paper, we suggest to improve the core behavior by using a three-parameter core model. This model is composed of flexible stiffness with a central shear layer. We will show that this core model is equivalent to a nonlocal elastic core model to account for nonlocal interactions between each beam. This structural modeling can also be viewed as the generalization of the recent study devoted to buckling of elastic column on nonlocal foundation. ${ }^{14}$ This nonlocal foundation was shown to be equivalent to Reissner's model. ${ }^{15}$ Reissner's model has been analyzed in the paper of $\operatorname{Kerr}^{16}$ and has been used for plate foundation or simplified beam foundation by Horvath ${ }^{17}$ or for Euler-Bernoulli beam models by Horvath. ${ }^{18}$ The bending behavior of Timoshenko beams on Reissner foundation was studied by Avramidis and Morfidis. ${ }^{19}$ Wang and Zhang ${ }^{20}$ recently used this three-parameter elastic foundation model for the analysis of adhesively bonded joints. The recent paper of di Paola et al. ${ }^{21}$ should also be mentioned for the presentation of a nonlocal foundation model based on an integral operator introduced from fractional derivative mathematical tools. Friswell et al. ${ }^{22}$ studied elastic beams on nonlocal elastic and viscoelastic foundation.

Elastically connected beam models have also been used as continuous systems models for carbon nanotubes (see for instance Refs. 23-28). The elastic layer 
provides a linear model for interatomic van der Waals forces. The nonlocal character of the constitutive behavior can be taken into account for each beam (see for instance Refs. 25, 27 and 28), but the nonlocal character of the interaction has not been previously considered. This paper presents some new results for the buckling of elastically connected beams with a nonlocal behavior for the strong interaction between the beams.

\section{Energy Equations}

The total potential energy of the axially loaded composite beams with a lateral nonlocal interaction can be formulated as a functional of the deflection variables:

$$
\begin{aligned}
U\left[w_{1}, w_{2}, w_{k}\right]= & \int_{0}^{L} \frac{1}{2} E I_{1} w_{1}^{\prime \prime 2} d x+\frac{1}{2} E I_{2} w_{2}^{\prime \prime 2}+\frac{1}{2} k_{1}\left(w_{1}-w_{k}\right)^{2}+\frac{1}{2} k_{2}\left(w_{2}-w_{k}\right)^{2} \\
& +\frac{1}{2} g w_{k}^{\prime 2} d x-\int_{0}^{L} \frac{1}{2} P_{1} w_{1}^{\prime 2}+\frac{1}{2} P_{2} w_{2}^{\prime 2} d x
\end{aligned}
$$

where $E I_{1}$ (or $E_{1} I_{1}$ ) is the flexural rigidity of one beam, and $E I_{2}$ (or $E_{2} I_{2}$ ) is the flexural rigidity of the other beam. The length of each beam is denoted by $L$. The three-parameter interaction model (or Reissner's model) consists of two linear elastic spring layers of constants $k_{1}$ and $k_{2}$, respectively, interconnected by a unit thickness shear layer of constant $g$ (Fig. 1). We will show that this model is a nonlocal interaction model. $w_{1}(x)$ is the deflection of one beam, $w_{2}(x)$ is the deflection of the second beam, and $w_{k}$ is the deflection of the shear layer. The composite beam is loaded by two independent load parameters $\left(P_{1}, P_{2}\right)$ acting on the centroidal axis of each beam. By taking the stationarity condition of the total potential energy $\delta U=0$ with respect to the assumed independent variables $\left(w_{1}, w_{2}, w_{k}\right)$, one obtains the coupled system of differential equations:

$$
\left\{\begin{array}{l}
E I_{1} w_{1}^{(4)}+P_{1} w_{1}^{\prime \prime}+k_{1}\left(w_{1}-w_{k}\right)=0 \\
E I_{2} w_{2}^{(4)}+P_{2} w_{2}^{\prime \prime}+k_{2}\left(w_{2}-w_{k}\right)=0 \\
\left(k_{1}+k_{2}\right) w_{k}-g w_{k}^{\prime \prime}=k_{1} w_{1}+k_{2} w_{2}
\end{array}\right.
$$

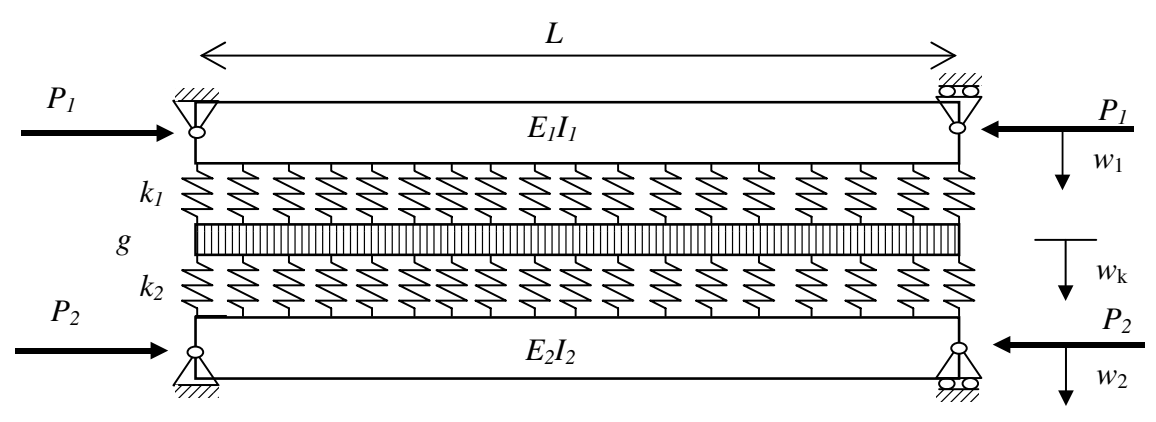

Fig. 1. Hinge-hinge nonlocally connected beams. 
with the boundary conditions:

$$
\begin{gathered}
{\left[\left(-E I_{1} w_{1}^{\prime \prime \prime}-P_{1} w_{1}^{\prime}\right) \delta w_{1}\right]_{0}^{L}=0, \quad\left[E I_{1} w_{1}^{\prime \prime} \delta w_{1}^{\prime}\right]_{0}^{L}=0,} \\
{\left[\left(-E I_{2} w_{2}^{\prime \prime \prime}-P_{2} w_{2}^{\prime}\right) \delta w_{2}\right]_{0}^{L}=0, \quad\left[E I_{2} w_{2}^{\prime \prime} \delta w_{2}^{\prime}\right]_{0}^{L}=0 \quad \text { and } \quad\left[g w_{k}^{\prime} \delta w_{k}\right]_{0}^{L}=0 .}
\end{gathered}
$$

The last differential equation of Eq. (2) can be presented in the following format:

$$
w_{k}-l_{c}^{2} w_{k}^{\prime \prime}=\frac{k_{1} w_{1}+k_{2} w_{2}}{k_{1}+k_{2}} \quad \text { with } l_{c}^{2}=\frac{g}{k_{1}+k_{2}} .
$$

Equation (4) shows that the deflection in the shear layer $w_{k}$ can be also expressed in an integral format of the average deflections from the introduction of an integral operator (see Ref. 29 and also Ref. 14):

$$
\bar{w}-l_{c}^{2} \bar{w}^{\prime \prime}=w \Rightarrow \bar{w}=\int_{0}^{L} G(x, y) w(y) d y .
$$

The weighting function $G(x, y)$ is the Green's function of the differential system with appropriate boundary conditions obtained from the variational principle (see Eq. (3)). Therefore, the differential equation clearly shows that the shear deflection $w_{k}(x)$ is a spatial weighted average of the deflection variables $w_{1}(x)$ and $w_{2}(x)$.

$$
w_{k}=\frac{\overline{k_{1} w_{1}+k_{2} w_{2}}}{k_{1}+k_{2}}=\frac{k_{1}}{k_{1}+k_{2}} \overline{w_{1}}+\frac{k_{2}}{k_{1}+k_{2}} \overline{w_{2}} .
$$

The mathematical problem can be also reduced to a system of two integrodifferential equations by inserting the nonlocal dependence of $w_{k}$ with respect to the beam deflections:

$$
\begin{gathered}
\left\{\begin{array}{l}
E I_{1} w_{1}^{(4)}+P_{1} w_{1}^{\prime \prime}+f_{1}\left[w_{1}, w_{2}\right]=0 \\
E I_{2} w_{2}^{(4)}+P_{2} w_{2}^{\prime \prime}+f_{2}\left[w_{2}, w_{1}\right]=0
\end{array}\right. \text { with } \\
\left\{\begin{array}{l}
f_{1}\left[w_{1}, w_{2}\right]=k_{1} w_{1}-k_{1}\left(\frac{k_{1}}{k_{1}+k_{2}} \overline{w_{1}}+\frac{k_{2}}{k_{1}+k_{2}} \overline{w_{2}}\right) \\
f_{2}\left[w_{1}, w_{2}\right]=k_{2} w_{2}-k_{2}\left(\frac{k_{1}}{k_{1}+k_{2}} \overline{w_{1}}+\frac{k_{2}}{k_{1}+k_{2}} \overline{w_{2}}\right) .
\end{array}\right.
\end{gathered}
$$

The nonlocal constitutive relationship can be also written in the following matrix form:

$$
\left.\left(\begin{array}{l}
f_{1} \\
f_{2}
\end{array}\right)=\left(\begin{array}{cc}
k_{1} & 0 \\
0 & k_{2}
\end{array}\right)\left(\begin{array}{l}
w_{1} \\
w_{2}
\end{array}\right)-\frac{1}{k_{1}+k_{2}} \quad \begin{array}{cc}
k_{1}^{2} & k_{1} k_{2} \\
k_{1} & k_{2}^{2}
\end{array}\right)\left(\begin{array}{l}
\overline{w_{1}} \\
\overline{w_{2}}
\end{array}\right)
$$

This model generalizes the one-dimensional nonlocal constitutive relationship considered by Challamel et al. ${ }^{14}$ for buckling on nonlocal foundation (namely $w_{2} \equiv 0$ in the present problem). It is easy to check that this nonlocal constitutive relationship is 
reduced to the usual local interaction when the characteristic length $l_{c}$ is vanishing:

$$
l_{c} \rightarrow 0 \Rightarrow\left(\begin{array}{c}
f_{1} \\
f_{2}
\end{array}\right)=k_{e q}\left(\begin{array}{c}
w_{1}-w_{2} \\
w_{2}-w_{1}
\end{array}\right) \quad \text { with } k_{e q}=\frac{k_{1} k_{2}}{k_{1}+k_{2}} .
$$

The system of three differential equations (2) can be reduced to a single equation dealing with one variable, for instance the deflection $w_{1}$. Considering the first equation of Eq. (2), the shear deflection $w_{k}$ can be expressed with respect to $w_{1}$ :

$$
w_{k}=w_{1}+\frac{P_{1}}{k_{1}} w_{1}^{\prime \prime}+\frac{E I_{1}}{k_{1}} w_{1}^{(4)} .
$$

The third equation of Eq. (2) leads to the mathematical expression of $w_{2}$ with respect to $w_{1}$ :

$$
w_{2}=w_{1}+\left(P_{1} \frac{k_{1}+k_{2}}{k_{1} \cdot k_{2}}-\frac{g}{k_{2}}\right) w_{1}^{\prime \prime}+\left(E I_{1} \frac{k_{1}+k_{2}}{k_{1} \cdot k_{2}}-g \frac{P_{1}}{k_{1} \cdot k_{2}}\right) w_{1}^{(4)}-g \frac{E I_{1}}{k_{1} \cdot k_{2}} w_{1}^{(6)} .
$$

The final 10th differential equation is finally obtained from the second equation of Eq. (2) with the consideration of Eqs. (10) and (11):

$$
A_{1} w_{1}^{(10)}+A_{2} w_{1}^{(8)}+A_{3} w_{1}^{(6)}+A_{4} w_{1}^{(4)}+A_{5} w_{1}^{\prime \prime}=0,
$$

with

$$
\begin{gathered}
A_{1}=-g \frac{E I_{1} E I_{2}}{k_{1} k_{2}}, \quad A_{2}=E I_{1} E I_{2} \frac{k_{1}+k_{2}}{k_{1} k_{2}}-\frac{g}{k_{1} k_{2}}\left(E I_{2} P_{1}+E I_{1} P_{2}\right), \\
A_{3}=\frac{k_{1}+k_{2}}{k_{1} k_{2}}\left(E I_{2} P_{1}+E I_{1} P_{2}\right)-g\left(\frac{E I_{1}}{k_{1}}+\frac{E I_{2}}{k_{2}}\right)-g \frac{P_{1} P_{2}}{k_{1} k_{2}} \\
A_{4}=E I_{1}+E I_{2}-g\left(\frac{P_{1}}{k_{1}}+\frac{P_{2}}{k_{2}}\right)+P_{1} P_{2} \frac{k_{1}+k_{2}}{k_{1} k_{2}} \text { and } A_{5}=P_{1}+P_{2}-g,
\end{gathered}
$$

with the 10 boundary conditions given by Eq. (3).

\section{Method of Solution}

We adopt the following nondimensional terms:

$$
\begin{gathered}
p_{1}=\frac{P_{1} L^{2}}{E I_{1}} ; \quad p_{2}=\frac{P_{2} L^{2}}{E I_{2}} ; \quad E I_{0}=E I_{1}+E I_{2} ; \quad \mu_{1}=\frac{E I_{1}}{E I_{0}} ; \quad \mu_{2}=\frac{E I_{2}}{E I_{0}} ; \\
\alpha^{2}=\frac{1}{l_{c}^{2}}=\frac{k_{1}+k_{2}}{g} ; \quad \alpha^{*}=\alpha L ; \quad k_{1}^{*}=\frac{k_{1} L^{4}}{E I_{1}} ; \quad k_{2}^{*}=\frac{k_{2} L^{4}}{E I_{2}} ; \\
g^{*}=\frac{g L^{2}}{E I_{0}} ; \quad \alpha^{* 2}=\frac{\mu_{1} k_{1}^{*}+\mu_{2} k_{2}^{*}}{g^{*}} ; \quad w^{*}=\frac{w}{L} ; \quad \text { and } \quad x^{*}=\frac{x}{L} .
\end{gathered}
$$


In view of the aforementioned terms, the 10th-order differential equation (12) may be written in a dimensionless format with dimensionless differentiation as:

$$
a_{1} w_{1}^{*(10)}+a_{2} w_{1}^{*(8)}+a_{3} w_{1}^{*(6)}+a_{4} w_{1}^{*(4)}+a_{5} w_{1}^{* \prime \prime}=0,
$$

with

$$
\begin{gathered}
a_{1}=1, \quad a_{2}=-\alpha^{* 2}+p_{1}+p_{2}, \quad a_{3}=k_{1}^{*}+k_{2}^{*}-\alpha^{* 2}\left(p_{1}+p_{2}\right)+p_{1} p_{2}, \\
a_{4}=-\frac{k_{1}^{*} k_{2}^{*}}{g^{*}}+p_{1} k_{2}^{*}+p_{2} k_{1}^{*}-\alpha^{* 2} p_{1} p_{2} \quad \text { and } \\
a_{5}=k_{1}^{*} k_{2}^{*}-\mu_{1} \frac{k_{1}^{*} k_{2}^{*}}{g^{*}} p_{1}-\mu_{2} \frac{k_{1}^{*} k_{2}^{*}}{g^{*}} p_{2} .
\end{gathered}
$$

By integrating Eq. (14) twice, one obtains an 8th-order differential equation:

$$
a_{1} w_{1}^{*(8)}+a_{2} w_{1}^{*(6)}+a_{3} w_{1}^{*(4)}+a_{4} w_{1}^{* \prime \prime}+a_{5} w_{1}^{*}=a_{5}\left(C_{9} x^{*}+C_{10}\right) .
$$

This differential equation is linear with constant coefficients, so that the solution can be assumed in the form:

$$
w_{1}^{*}=\sum_{i=1}^{8} C_{i} e^{\lambda_{i} x^{*}}+C_{9} x^{*}+C_{10} .
$$

The substitution of this solution into the 8th-order differential equation furnishes the auxiliary equation as follows:

$$
a_{1} \lambda^{8}+a_{2} \lambda^{6}+a_{3} \lambda^{4}+a_{4} \lambda^{2}+a_{5}=0 .
$$

The above equation is a quartic in $z=\lambda^{2}$, namely

$$
a_{1} z^{4}+a_{2} z^{3}+a_{3} z^{2}+a_{4} z+a_{5}=0 .
$$

This quartic equation can be exactly analytically solved by using Ferrari or Cardano's method. In this paper, we present some analytical solutions for some specific boundary conditions, namely the pinned-pinned case.

\section{Closed-Form Solutions}

For the pinned-pinned case, the following boundary conditions are obtained from the variational principle:

$$
\begin{aligned}
& w_{1}(0)=w_{1}^{\prime \prime}(0)=w_{1}(L)=w_{1}^{\prime \prime}(L)=0 \quad \text { and } \\
& w_{2}(0)=w_{2}^{\prime \prime}(0)=w_{2}(L)=w_{2}^{\prime \prime}(L)=0
\end{aligned}
$$

with the higher-order boundary conditions expressed as:

$$
\begin{gathered}
w_{k}(0)=w_{k}(L)=0 \\
w_{k}^{\prime}(0)=w_{k}(L)=0 \quad \text { or } \quad w_{k}(0)=w_{k}^{\prime}(L)=0 \\
w_{k}^{\prime}(0)=w_{k}^{\prime}(L)=0 .
\end{gathered}
$$


A simple closed-form solution can be provided in the first case of these higher-order boundary condition Eq. (19a), based on a complete sinusoidal basis of the deflection. This higher-order boundary condition corresponds to the case of strong adhesion of the foundation material to the rigid wall $\left(\operatorname{Kerr}^{16}\right)$. It is then possible to take a sinusoidal buckling shape written as:

$$
w_{1}^{*}\left(x^{*}\right)=w_{0}^{*} \sin \left(n \pi x^{*}\right),
$$

verifying the correct boundary conditions, and leading to the exact buckling load boundary:

$$
\begin{gathered}
f\left(p_{1}, p_{2}\right)=b_{1} p_{1} p_{2}+b_{2} p_{1}+b_{3} p_{2}+b_{4}=0 \quad \text { with } \\
b_{1}=(n \pi)^{4}+\alpha^{* 2}(n \pi)^{2}, \quad b_{2}=-(n \pi)^{6}-\alpha^{* 2}(n \pi)^{4}-k_{2}^{*}(n \pi)^{2}-\mu_{1} \frac{k_{1}^{*} k_{2}^{*}}{g^{*}}, \\
b_{3}=-(n \pi)^{6}-\alpha^{* 2}(n \pi)^{4}-k_{1}^{*}(n \pi)^{2}-\mu_{2} \frac{k_{1}^{*} k_{2}^{*}}{g^{*}} \text { and } \\
b_{4}=(n \pi)^{8}+\alpha^{* 2}(n \pi)^{6}+\left(k_{1}^{*}+k_{2}^{*}\right)(n \pi)^{4}+\frac{k_{1}^{*} k_{2}^{*}}{g^{*}}(n \pi)^{2}+k_{1}^{*} k_{2}^{*} .
\end{gathered}
$$

Therefore, the exact buckling load boundary in the loading space $\left(p_{1}, p_{2}\right)$ is expressed as:

$$
p_{1}=-\frac{b_{3} p_{2}+b_{4}}{b_{1} p_{2}+b_{2}} \quad \text { if } p_{2} \neq-\frac{b_{2}}{b_{1}}, \quad \text { or } \quad p_{2}=-\frac{b_{2} p_{1}+b_{4}}{b_{1} p_{1}+b_{3}} \quad \text { if } p_{1} \neq-\frac{b_{3}}{b_{1}} .
$$

The boundary of the stability domain in the loading space is shown in Figs. 2 and 3. The maximum buckling load in each direction is obtained for:

$$
\frac{\partial f}{\partial p_{i}}=0
$$

leading to

$$
\begin{aligned}
& \hat{p}_{1}=-\frac{b_{3}}{b_{1}}=\frac{(n \pi)^{6}+\alpha^{* 2}(n \pi)^{4}+k_{1}^{*}(n \pi)^{2}+\mu_{2} \frac{k_{1}^{*} k_{2}^{*}}{g^{*}}}{(n \pi)^{4}+\alpha^{* 2}(n \pi)^{2}} \text { and } \\
& \hat{p}_{2}=-\frac{b_{2}}{b_{1}}=\frac{(n \pi)^{6}+\alpha^{* 2}(n \pi)^{4}+k_{2}^{*}(n \pi)^{2}+\mu_{1} \frac{k_{1}^{*} k_{2}^{*}}{g^{*}}}{(n \pi)^{4}+\alpha^{* 2}(n \pi)^{2}} .
\end{aligned}
$$

These values are the positive asymptotic values in each direction, for a coupled dimensionless buckling load which tends toward an infinite negative value-tension case (see Figs. 2 and 3). It is worth mentioning that the stability domain has the convexity property in the loading space, a property that can be referred to the wellknown Papkovitch-Schaefer theorem (see the applications of this theorem for outof-plane buckling problem ${ }^{30-32}$ ). 


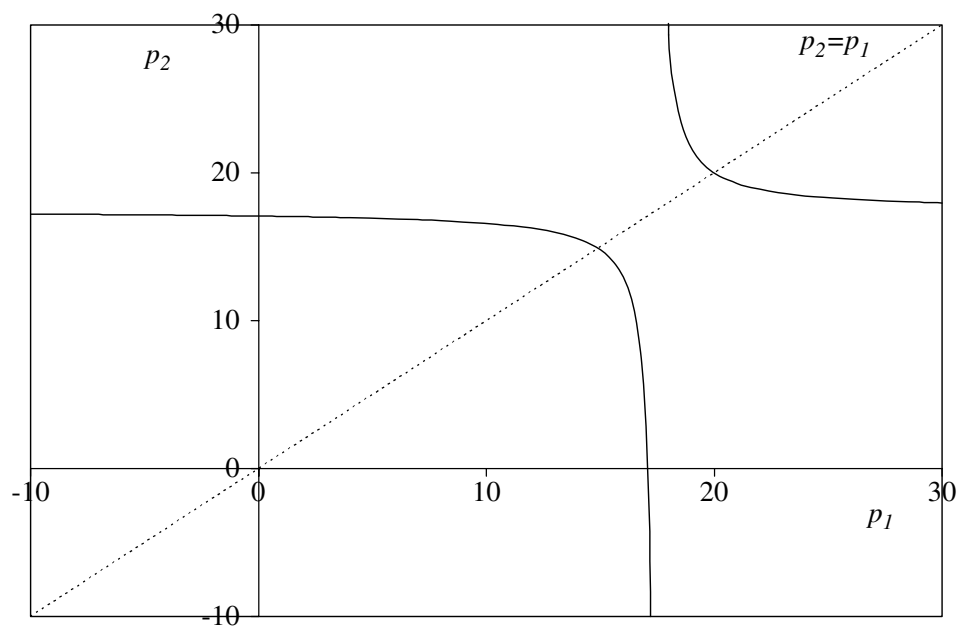

Fig. 2. Stability domain in the space of loading parameters $\left(p_{1}, p_{2}\right)$; symmetrical case; $\mu_{1}=\mu_{2}=\frac{1}{2}$; $k_{1}^{*}=k_{2}^{*}=100 ; g^{*}=10$.

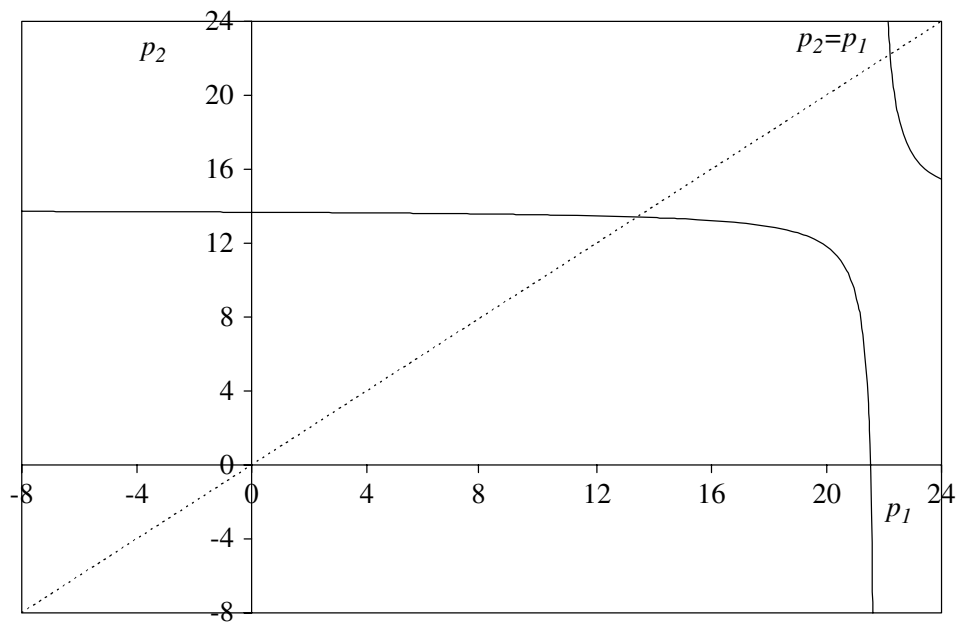

Fig. 3. Stability domain in the space of loading parameters $\left(p_{1}, p_{2}\right) ;$ unsymmetrical case; $\mu_{1}=\frac{1}{4} ; \mu_{2}=\frac{3}{4}$; $k_{1}^{*}=150 ; k_{2}^{*}=50 ; g^{*}=10$.

\section{Buckling Cases}

The symmetrical configuration with symmetrical boundary conditions is now studied for the specific structural parameters:

$$
P_{1}=P_{2}=\frac{P_{0}}{2}, \quad E I_{1}=E I_{2}=\frac{E I_{0}}{2} \quad \text { and } \quad k_{1}=k_{2}=\frac{k_{0}}{2} .
$$


The dimensionless parameters of the problem are then reduced to:

$$
\begin{gathered}
p_{0}=\frac{P_{0} L^{2}}{E I_{0}}, \quad k_{0}^{*}=\frac{k_{0} L^{4}}{E I_{0}}, \quad \alpha^{2}=\frac{1}{l_{c}^{2}}=\frac{k_{0}}{g} ; \quad \alpha^{*}=\alpha L ; \\
g^{*}=\frac{g L^{2}}{E I_{0}} ; \quad w^{*}=\frac{w}{L} \quad \text { and } \quad x^{*}=\frac{x}{L} .
\end{gathered}
$$

For symmetrical reasons, the buckling mode is symmetrical or antisymmetrical in this configuration.

\subsection{Antisymmetrical case}

The antisymmetrical case is first considered, based on:

$$
w_{1}=w_{2}=w
$$

The differential equations (2) are then reduced to a system of two differential equations:

$$
\left\{\begin{array}{l}
E I_{0} w^{(4)}+P_{0} w^{\prime \prime}+k_{0}\left(w-w_{k}\right)=0 \\
w_{k}-l_{c}^{2} w_{k}^{\prime \prime}=w
\end{array} \quad \text { with } l_{c}=\frac{g}{k_{0}} .\right.
$$

The second differential equation clearly shows that the deflection of the shear layer is exactly equal to the nonlocal deflection, according to Eringen's differential model. ${ }^{29}$ Finally, the sixth-order differential equation is obtained from:

$$
-g \frac{E I_{0}}{k_{0}} w^{(6)}+\left(E I_{0}-g \frac{P_{0}}{k_{0}}\right) w^{(4)}+\left(P_{0}-g\right) w^{\prime \prime}=0 .
$$

The closed-form solution for the pinned-pinned case is written as:

$$
p_{0}=\frac{(n \pi)^{4}+\alpha^{* 2}(n \pi)^{2}+\alpha^{* 2} g^{*}}{(n \pi)^{2}+\alpha^{* 2}} .
$$

The "local" constitutive law is obtained for a vanishing length scale $l_{c}$ leading to the buckling load $p_{0}=(n \pi)^{2}$. Equation (30) clearly shows that the buckling load is affected by the nonlocal character of the elastic connection law. The two asymptotic cases correspond to the "local" noncomposite beam, and the full composite beam:

$$
\lim _{\alpha^{*} \rightarrow 0} p_{0}=(n \pi)^{2} \quad \text { and } \quad \lim _{\alpha^{*} \rightarrow \infty} p_{0}=(n \pi)^{2}+g^{*} .
$$

The transition from the "local" noncomposite beam to the full composite beam is shown in Fig. 4. For sufficiently small values of the dimensionless shear stiffness $g^{*}$, the fundamental buckling mode is obtained for $n=1$ (no phenomenon of mode exchange). Note that the buckling load of the full composite beam $\left(\alpha^{*} \rightarrow \infty\right)$ is finite and is expressed in terms of the buckling load of the noncomposite beam corrected by the shear stiffness (see Eq. (31)). Such kind of behaviors is generally observed for composite beams with interlayer slip (see for instance Ref. 33). Furthermore, when the shear stiffness $g^{*}$ is increasing, the phenomenon of buckling mode exchange 


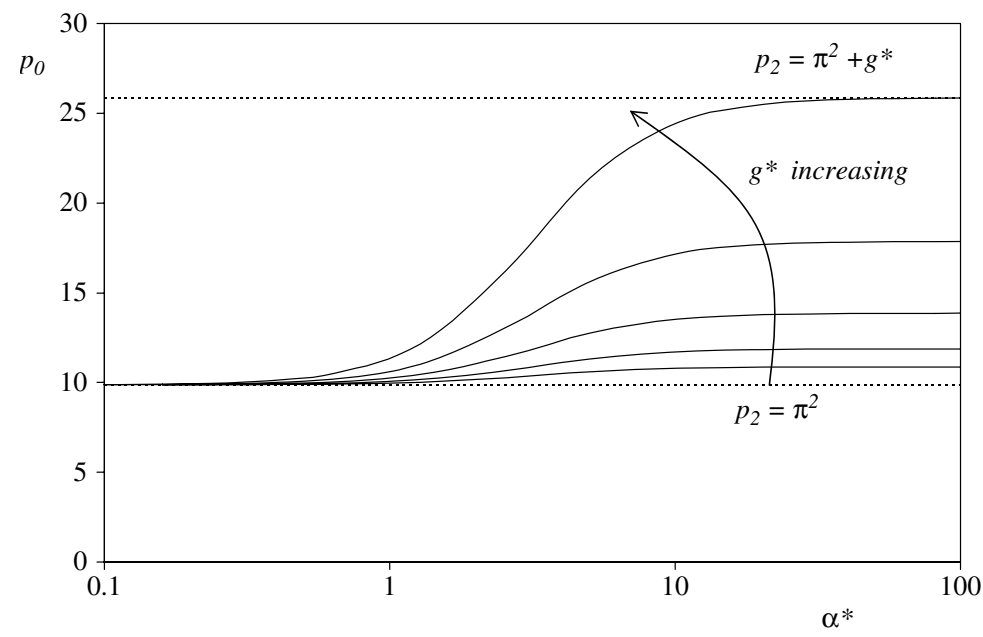

Fig. 4. Buckling load with respect to the connection parameter $\alpha^{*} ; g^{*} \in\{1 ; 2 ; 4 ; 8 ; 16\}$; fundamental (antisymmetrical) buckling mode; $n=1$.

appears (see also Ref. 34 for the out-of-plane behavior of composite beams). This is clearly highlighted in Fig. 5. This phenomenon appears for the dimensionless shear layer stiffness approximately equal to $g^{*}=90$ (Fig. 6).

\subsection{Symmetrical case}

The symmetrical case is based on:

$$
w_{1}=-w_{2}=w \quad \text { and } \quad w_{k}=0 .
$$

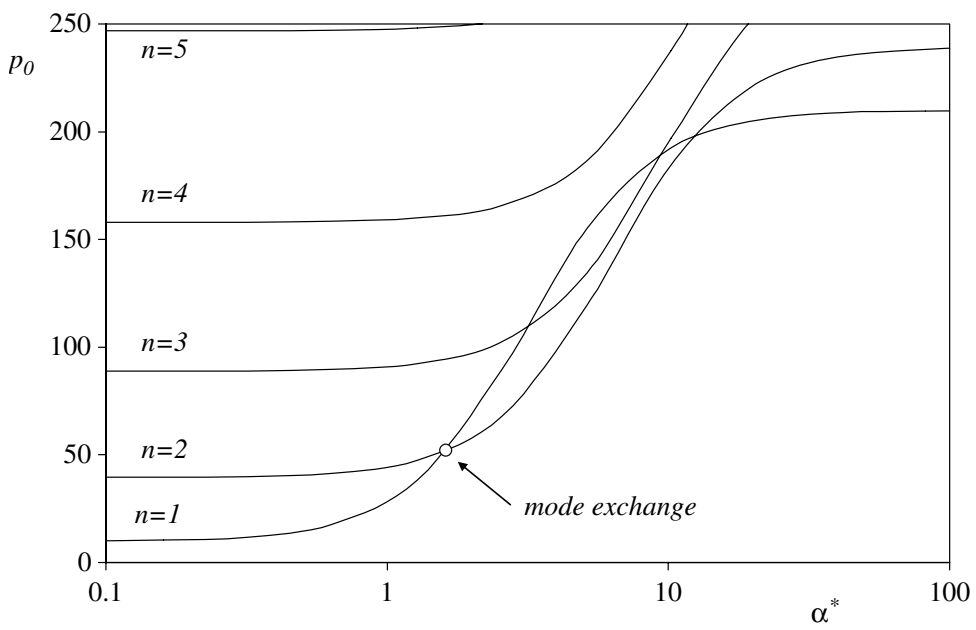

Fig. 5. Phenomenon of mode exchange of the (antisymmetrical) buckling mode for sufficiently large stiffness connection parameter $\alpha^{*} ; g^{*}=200$. 


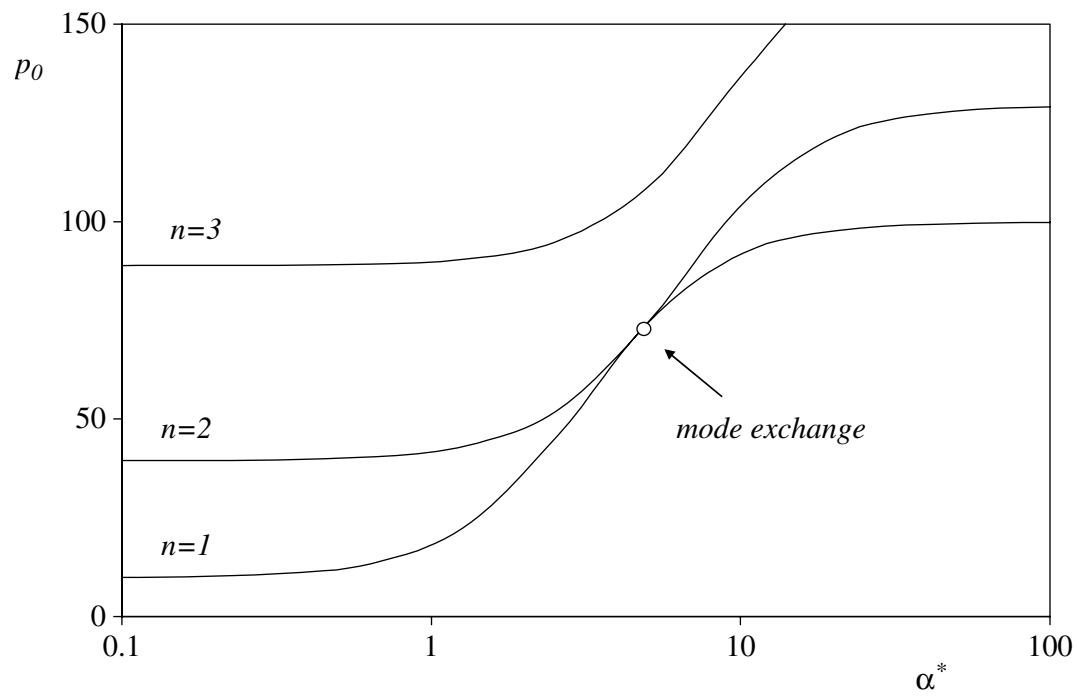

Fig. 6. Phenomenon of mode exchange of the (antisymmetrical) buckling mode for sufficiently large stiffness connection parameter $\alpha^{*} ; g^{*}=90$.

One recognizes the buckling differential equation of a beam on Winkler foundation as:

$$
E I_{0} w^{(4)}+P_{0} w^{\prime \prime}+k_{0} w=0
$$

whose buckling load is given for the pinned-pinned case as:

$$
p_{0}=(n \pi)^{2}+\frac{k_{0}^{*}}{(n \pi)^{2}} \quad \text { with } k_{0}^{*}=\alpha^{* 2} g^{*} .
$$

The comparison between the symmetrical and the antisymmetrical buckling mode is shown in Fig. 7. The antisymmetrical buckling load leads to smaller buckling load, compared to the symmetrical case, which can be easily explained from the following inequality:

$$
\begin{aligned}
p_{0}^{(A S)} & =\frac{(n \pi)^{4}+\alpha^{* 2}(n \pi)^{2}+\alpha^{* 2} g^{*}}{(n \pi)^{2}+\alpha^{* 2}} \\
& =(n \pi)^{2}+\frac{\alpha^{* 2} g^{*}}{(n \pi)^{2}+\alpha^{* 2}}<(n \pi)^{2}+\frac{\alpha^{* 2} g^{*}}{(n \pi)^{2}}=p_{0}^{(S)} .
\end{aligned}
$$

Note, however, that the symmetrical buckling load has no asymptotic limit in case of infinite value of the connection parameter $\alpha^{*}$. The symmetric and antisymmetrical buckling values are highlighted in Fig. 2 from the intersection of the symmetric axis $p_{1}=p_{2}$ with the buckling load boundary. 


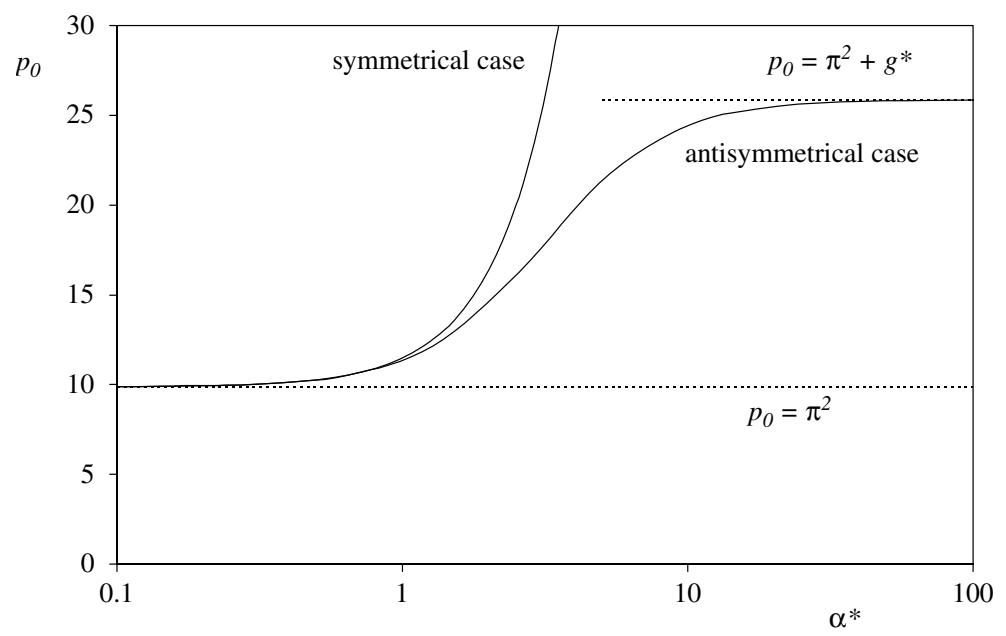

Fig. 7. Comparison of the buckling load for the symmetrical and the antisymmetrical case; $g^{*}=16$.

\section{Nonlocal Eringen's Law and Other General Nonlocal's Laws}

The general nonlocal connection law can be also considered:

$$
\begin{gathered}
\left(\begin{array}{l}
f_{1} \\
f_{2}
\end{array}\right)=k_{e q}\left\{\zeta\left(\begin{array}{c}
w_{1}-w_{2} \\
w_{2}-w_{1}
\end{array}\right)+(1-\zeta)\left(\frac{\overline{w_{1}-w_{2}}}{w_{2}-w_{1}}\right)\right\} \quad \text { with } \zeta=\left(\frac{a}{l_{c}}\right)^{2} \\
\text { and } k_{e q}=\frac{k_{1} k_{2}}{k_{1}+k_{2}} .
\end{gathered}
$$

The case $a=l_{c}$, or equivalently $\zeta=1$ leads to the local beam elastic interaction given by Eq. (9). Such a combination of local and nonlocal variables has been already used for beam nonlocal elastic model, ${ }^{35,36}$ dynamics of heterogeneous dispersive materials, ${ }^{37}$ or inelastic nonlocal softening media. ${ }^{38-40}$ Note that the nonlocal elastic connection law can be also expressed in a differential format as:

$$
\left\{\begin{array}{l}
f_{1}-l_{c}^{2} f_{1}^{\prime \prime}=k_{e q}\left(\Delta w_{1}-a^{2} \Delta w_{1}^{\prime \prime}\right) \\
f_{2}-l_{c}^{2} f_{2}^{\prime \prime}=k_{e q}\left(\Delta w_{2}-a^{2} \Delta w_{2}^{\prime \prime}\right)
\end{array} \quad \text { with } \Delta w_{1}=w_{1}-w_{2}=-\Delta w_{2} .\right.
$$

Such a nonlocal model comprises the purely nonlocal connection model $(a=0)$, and the gradient elasticity connection model $\left(l_{c}=0\right)$. This nonlocal model can be derived from application of the variational principle written as:

$$
\begin{aligned}
U\left[w_{1}, w_{2}, \Delta \bar{w}_{1}\right]= & \int_{0}^{L} \frac{1}{2} E I_{1} w_{1}^{\prime \prime 2} d x+\frac{1}{2} E I_{2} w_{2}^{\prime \prime 2}+\frac{1}{2} k_{e q}(2 \zeta-1)\left(\Delta w_{1}\right)^{2} \\
& +\frac{1}{2} k_{e q}(1-\zeta)\left(\Delta w_{1}-\Delta \bar{w}_{1}\right)^{2}+\frac{1}{2} k_{e q}(1-\zeta) l_{c}^{2}\left(\Delta \bar{w}_{1}^{\prime}\right)^{2} d x \\
& -\int_{0}^{L} \frac{1}{2} P_{1} w_{1}^{\prime 2}+\frac{1}{2} P_{2} w_{2}^{\prime 2} d x,
\end{aligned}
$$


leading to the coupled system of the following differential equations:

$$
\left\{\begin{array}{l}
E I_{1} w_{1}^{(4)}+P_{1} w_{1}^{\prime \prime}+k_{e q}\left[\zeta \Delta w_{1}+(1-\zeta) \Delta \bar{w}_{1}\right]=0 \\
E I_{2} w_{2}^{(4)}+P_{2} w_{2}^{\prime \prime}+k_{e q}\left[\zeta \Delta w_{2}+(1-\zeta) \Delta \bar{w}_{2}\right]=0 \\
\Delta \bar{w}_{1}-l_{c}^{2} \Delta \bar{w}_{1}^{\prime \prime}=\Delta w_{1} ; \Delta w_{2}=-\Delta w_{1} .
\end{array}\right.
$$

The symmetrical configuration (with symmetrical boundary conditions) is now studied for the specific structural parameters:

$$
P_{1}=P_{2}=\frac{P_{0}}{2}, \quad E I_{1}=E I_{2}=\frac{E I_{0}}{2} .
$$

For symmetrical reasons, the buckling mode is symmetrical or antisymmetrical in this configuration. The antisymmetrical case is first considered, based on:

$$
w_{1}=w_{2}=w
$$

leading to:

$$
E I_{0} w^{(4)}+P_{0} w^{\prime \prime}=0 .
$$

Therefore, the symmetrical mode is not influenced by some beam interaction influences, neither nonlocal scale effects. The symmetrical mode is now considered, based on:

$$
w_{1}=-w_{2}=w .
$$

One recognizes the buckling differential equation of a beam on Reissner foundation $^{14}$ as:

$$
E I_{0} w^{(4)}+P_{0} w^{\prime \prime}+4 k_{e q}[\zeta w+(1-\zeta) \bar{w}]=0 .
$$

This problem has been already treated in Ref. 14, and such a symmetrical buckling mode implicitly contains some scale effects. However, the fundamental symmetrical buckling load is larger than the fundamental antisymmetrical buckling load that does not contain any scale effects, and one can conclude that such a nonlocal modeling has some apparent deficiencies from the inherent scale effect property (at least for the symmetrical nonlocal connected columns).

\section{Discussion on Shear Columns, Timoshenko Theory as a Nonlocal Theory}

This part is devoted to the Timoshenko theory that can be now understood as a nonlocal theory. It is now acted that Timoshenko beam theory can be generalized to a nonlocal framework using Eringen's nonlocal elastic law. ${ }^{41,42} \mathrm{Ma}$ et al. $^{43}$ also developed a Timoshenko model based on a modified couple stress theory that can capture the stiffening effect at small length scales. However, the analysis of the local Timoshenko model as a nonlocal theory has not been yet presented, to the authors' knowledge. In the presence of axial loads, Engesser and Haringx's type models have 
to be distinguished. ${ }^{44} \mathrm{~A}$ discussion about the hyperelastic formulation of generic Timoshenko models can be found in Attard and Hunt. ${ }^{2}$

The energy functional of Engesser-type column is given for this problem by:

$$
U[\psi, w]=\int_{0}^{L} \frac{1}{2} E I \psi^{\prime 2}+\frac{\kappa G A}{2}\left(w^{\prime}-\psi\right)^{2}-\frac{1}{2} P w^{\prime 2} d x,
$$

where $\psi$ is the rotation; $G$ is the shear modulus; $A$ is the total area; and $\kappa$ is the so-called shear coefficient, a dimensionless factor. The stationarity of the energy functional $\delta U=0$ leads in this case to:

$$
\left\{\begin{array}{l}
-\kappa G A\left(w^{\prime \prime}-\psi^{\prime}\right)+P w^{\prime \prime}=0 \\
E I \psi^{\prime \prime}+\kappa G A\left(w^{\prime}-\psi\right)=0
\end{array},\right.
$$

with the natural boundary conditions:

$$
\left[E I \psi^{\prime} \delta \psi\right]_{0}^{L}=0 \quad \text { and } \quad\left[\left(-E I \psi^{\prime \prime}-P w^{\prime}\right) \delta w\right]_{0}^{L}=0 .
$$

The characteristic length $l_{c}$ can be introduced as:

$$
l_{c}=\sqrt{\frac{E I}{\kappa G A}} .
$$

The system of differential equations is now written with such a characteristic length as:

$$
\left\{\begin{array}{l}
-E I\left(w^{\prime \prime}-\psi^{\prime}\right)+P l_{c}^{2} w^{\prime \prime}=0 \\
-l_{c}^{2} \psi^{\prime \prime}=w^{\prime}
\end{array}\right.
$$

The second equation shows that the rotation is the nonlocal spatial average variable of the slope angle $w^{\prime}$ :

$$
=\overline{w^{\prime}} \quad \text { with } \overline{w^{\prime}}-l_{c}^{2} \overline{w^{\prime}}=w^{\prime} .
$$

The Timoshenko theory is clearly a nonlocal theory which can be expressed from the energy functional of one variable:

$$
U[w]=\int_{0}^{L} \frac{1}{2} E I\left(\overline{w^{\prime}}\right)^{2}+\frac{\kappa G A}{2}\left(w^{\prime}-\overline{w^{\prime}}\right)^{2}-\frac{1}{2} P w^{\prime 2} d x .
$$

Furthermore, Eq. (49) leads to the uncoupled differential equation:

$$
\left(E I-P l_{c}^{2}\right) \psi^{\prime \prime \prime}+P \psi^{\prime}=0 \quad \text { or } \quad\left(E I-P l_{c}^{2}\right) w^{(4)}+P w^{\prime \prime}=0 \quad \text { with } l_{c}=\sqrt{\frac{E I}{\kappa G A}},
$$

leading to the well-known Engesser formulae for most boundary conditions except for instance the fixed-pinned conditions ${ }^{45,46}$ :

$$
P^{T}=\frac{P^{E}}{1+\frac{P E}{\kappa G A}}=\frac{P^{E}}{1+\frac{P E}{E I} l_{c}^{2}} .
$$


For instance, for the pinned-pinned case, the Engesser formulae are simplified in the following equation:

$$
\frac{P^{T}}{P^{E}}=\frac{1}{1+\pi^{2}\left(\frac{l_{c}}{L}\right)^{2}} \quad \text { with } P^{E}=E I\left(\frac{\pi}{L}\right)^{2}
$$

These are also the formulae of the in-plane and out-of-plane buckling problems of Euler-Bernoulli beam model with Eringen's nonlocal law (see for instance Ref. 47). In fact, the nonlocal Euler-Bernoulli constitutive law is written as:

$$
M-l_{c}^{2} M^{\prime \prime}=E I \chi \quad \text { or equivalently } M=E I \bar{\chi} \quad \text { with } \bar{\chi}-l_{c}^{2} \bar{\chi}^{\prime \prime}=\chi,
$$

with $\chi$ as the curvature (one can choose $\chi=-w^{\prime \prime}$ ). This nonlocal constitutive law can be also presented in an integral format using the Green's operator of Eq. (5):

$$
M(x)=\int_{0}^{L} G(x, y) \chi(y) d y .
$$

The elastic energy of the nonlocal Euler-Bernoulli column can be expressed in the following form ${ }^{35,36}$ :

$$
U[w]=\int_{0}^{L} \frac{1}{2} E I \chi \bar{\chi}-\frac{1}{2} P w^{\prime 2} d x,
$$

Equation (57) is also equivalent to the double integral representation of the nonlocal beam problem, generally considered for integral nonlocal elasticity problems:

$$
U[w]=\int_{0}^{L} \int_{0}^{L} \frac{1}{2} E I G(x, y) \chi(x) \chi(y) d x d y-\int_{0}^{L} \frac{1}{2} P w^{\prime 2} d x,
$$

which is also equivalent to:

$$
U[w]=\int_{0}^{L} \frac{1}{2} M \chi-\frac{1}{2} P w^{\prime 2} d x \quad \text { with } M=E I \bar{\chi} .
$$

The energy functional is also shown to be equivalent to the simplified expression in case of Eringen's model ${ }^{47}$ :

$$
U[w]=\int_{0}^{L} \frac{1}{2}\left(E I-P l_{c}^{2}\right) w^{\prime \prime 2}-\frac{1}{2} P w^{\prime 2} d x .
$$

The differential equation of the buckling of nonlocal Euler-Bernoulli column is obtained from the application of variational principle and is given for instance by Zhang et al. ${ }^{36}$ :

$$
\left(E I-P l_{c}^{2}\right) w^{(4)}+P w^{\prime \prime}=0,
$$

which is exactly the differential equation of the Timoshenko column (see Eq. 52). Zhang et al. ${ }^{36}$ already mentioned the analogy between the vibration problems of nonlocal Euler-Bernoulli beams and the vibration problems of Rayleigh beams. It is 
shown in the present paper that the buckling problem of Timoshenko column is analogous to one of the nonlocal Euler-Bernoulli columns from the analogous differential equations. Therefore, Timoshenko buckling formulae will be strictly equivalent to nonlocal Euler-Bernoulli buckling formulae if the same boundary conditions at the beam extremity are equivalent.

Finally, we would like to discuss the Haringx-type formulae from the nonlocal point of view. The energy functional of Haringx-type column is given by:

$$
U=\int_{0}^{L} \frac{1}{2} E I \psi^{\prime 2}+\frac{\kappa G A+P}{2}\left(w^{\prime}-\psi\right)^{2}-\frac{1}{2} P w^{\prime 2} d x .
$$

The stationarity of the energy functional $\delta U=0$ furnishes:

$$
\left\{\begin{array}{l}
-\kappa G A w^{\prime \prime}+(\kappa G A+P) \psi^{\prime}=0 \\
E I \psi^{\prime \prime}+(\kappa G A+P)\left(w^{\prime}-\psi\right)=0
\end{array}\right.
$$

and leads to the nonlocal loading-dependent constitutive law:

$$
-l_{c}^{* 2} \psi^{\prime \prime}=w^{\prime} \quad \text { with } l_{c}^{*}=\sqrt{\frac{E I}{\kappa G A+P}} .
$$

In this case, the rotation $\psi$ is still a nonlocal spatial average variable of the slope angle $w^{\prime}$ but the "characteristic" length $l_{c}^{*}$ is no more material-dependent but also loading-dependent. Furthermore, the differential equations of Haringx-type column can be presented with a single variable as:

$$
E I \psi^{\prime \prime \prime}+P\left(1+\frac{P}{\kappa G A}\right)^{\prime}=0 \quad \text { or } \quad E I w^{(4)}+P\left(1+\frac{P}{\kappa G A}\right) w^{\prime \prime}=0 .
$$

This last differential equation can be also presented in the differential equation of the nonlocal problem:

$$
\left(E I-P l_{c}^{* 2}\right) w^{(4)}+P w^{\prime \prime}=0 \quad \text { with } l_{c}^{*}=\sqrt{\frac{E I}{\kappa G A+P}},
$$

leading to Haringx-type formulae ${ }^{1}$ :

$$
\left.P^{T}=\frac{\kappa G A}{2} \quad \sqrt{1+4 \frac{P_{E}}{\kappa G A}}-1\right) .
$$

\section{Concluding Remarks}

The buckling of an axially loaded elastic composite beam with a nonlocal core or a nonlocal connection system has been studied in this paper. The analytical results presented in this paper generalize the results recently published on the buckling of homogeneous columns on nonlocal elastic foundation. ${ }^{14}$ The composite beam or the sandwich beam is composed of two Euler-Bernoulli beams with a nonlocal elastic interaction. This nonlocal interaction is physically based on the Reissner's model 
based on three-parameters' interaction function. The connection model can be expressed in an integral format, using Eringen's differential equation, ${ }^{29}$ thereby inducing the nonlocal character of this beam elastic interaction model. The buckling solution is sensitive to some scale effects inherently contained in the nonlocal elastic law.

Some closed-form solutions of the buckling loads are given for the case of pinned-pinned boundary conditions. The correspondence between the buckling of the nonlocal composite column and the shear composite column is discussed. It is shown that the buckling behavior of this nonlocal composite column is close to one of the sandwich beams or composite beam-columns with interlayer slip. Finally, it is shown that the Timoshenko beam model is a nonlocal integral Euler-Bernoulli model. The scale effects are specifically highlighted for this nonlocal model.

The extension of such a beam interaction model, including the general stabilitydynamics coupling, is envisaged for general boundary conditions. The generalization of the nonlocal transversal interaction to a coupling with some shear interaction could be also studied to complete the interaction kinematics. The applications of such study can be also found for RC beams strengthened with composite laminates (see for instance Ref. 20,48). The kinematics of the adhesive layer is generally considered to play a crucial role from the global structural analysis: the physically motivated nonlocal model presented in this paper may be used for the understanding of the adhesive layer behavior, without ad-hoc assumptions relating the deflection in the shear layer from the deflections of the two other subelements.

\section{References}

1. Z. P. Bažant and L. Cedolin, Stability of Structures - Elastic, Inelastic, Fracture, and Damage Theories (Dover Publications, Inc., New-York, 2003).

2. M. M. Attard and J. Hunt, Column buckling with shear deformations - a hyperelastic formulation, International Journal of Solids Structures 45 (2008) 4322-4339.

3. J. Blaauwendraad, Shear in structural stability: On the Engesser-Haringx discord, Journal of Applied Mechanics 77 (2010) 031005.

4. C. M. Wang, J. N. Reddy and K. H. Lee, Shear Deformable Beams and Plates: Relationships with Classical Solutions (Elsevier, Oxford, UK, 2000).

5. C. B. Norris, W. S. Ericksen and W. J. Kommers, Flexural Rigidity of a Rectangular Strip of Sandwich Construction - Comparison between Mathematical Analysis and Results of Tests, Tech. Rep. Report 1505A (Forest Prod. Lab., Madison, WI, 1952).

6. N. J. Hoff, The Analysis of Structures (John Wiley and Sons, London, England, 1956).

7. F. J. Plantema, Sandwich Construction (John Wiley \& Sons, New York, 1966).

8. H. G. Allen, Analysis and Design of Structural Sandwich Panels (Pergamon Press, Oxford, 1969).

9. M. M. Attard and J. Hunt, Sandwich column buckling - a hyperelastic formulation, International Journal of Solids Structures 45 (2008) 5540-5555.

10. Y. Frostig, Elastica of sandwich panels with a transversely flexible core - a high-order theory approach, International Journal of Solids Structures 46 (2009) 2043-2059.

11. N. J. Hoff and S. E. Mautner, Bending and buckling of sandwich beams, Journal of the Aeronautical Sciences 15 (1948) 707. 
12. Y. Frostig, M. Baruch, O. Vilnay and I. Sheinman, A high-order theory for the bending of sandwich beams with a flexible core, Journal of Engineering Mechanics 118(5) (1992) 1026-1043.

13. Y. Frostig and M. Baruch, Buckling of simply-supported sandwich beams with transversely flexible core - a high order theory, Journal of Engineering Mechanics 119(3) (1993) 476-495.

14. N. Challamel, S. A. Meftah and F. Bernard, Buckling of elastic beams on nonlocal foundation: A revisiting of Reissner model, Mechanics Research Communications 37 (2010) 472-475.

15. E. Reissner, A note on deflections of plates on a viscoelastic foundation, Journal of Applied Mechanics 25 (1958) 144-145.

16. A. D. Kerr, Elastic and viscoelastic foundation models, Journal of Applied Mechanics 31 (1964) 491-498.

17. J. S. Horvath, New subgrade model applied to mat foundations, Journal of the Geotechnical Engineering, ASCE 109(12) (1983) 1567-1587.

18. J. S. Horvath, Beam-column analogy model for soil-structure interaction analysis, Journal of the Geotechnical Engineering 119(2) (1993) 358-364.

19. I. E. Avramidis and K. Morfidis, Bending of beams on three-parameter elastic foundation, International Journal of Solids Structures 43 (2006) 357-375.

20. J. Wang and C. Zhang, Three parameter, elastic foundation model for analysis of adhesively bonded joints, International Journal of Adhesion Adhesives 29 (2009) 495-502.

21. M. di Paola, F. Marino and M. Zingales, A generalized model of elastic foundation based on long-range interactions: integral and fractional model, International Journal of Solids Structures 46(17) (2009) 3124-3137.

22. M. I. Friswell, S. Adhikari and Y. Lei, Finite element analysis of beams with nonlocal foundations, International Journal for Numerical Methods in Engineering 71(11) (2007) $1365-1386$

23. C. Q. Ru, Degraded axial buckling strain of multiwalled carbon nanotubes due to interlayer slips, Journal of Applied Physics 89 (2000) 3426-3433.

24. C. Q. $\mathrm{Ru}$, Column buckling of multiwalled carbon nanotube with interlayer radial displacements, Physics Review B 62 (2002) 16962-16967.

25. S. Adali, Variational principles for multi-walled carbon nanotubes undergoing buckling based on nonlocal elasticity theory, Physics Letters A 372 (2008) 5701-5705.

26. S. G. Kelly and S. Srinivas, Free vibrations of elastically connected stretched beams, Journal of Sound and Vibration 326 (2009) 883-893.

27. K. Amara, A. Tounsi, I. Mechab and E. A. Adda-Bedia, Nonlocal elasticity effect on column buckling of multiwalled carbon nanotubes under temperature field, Applied Mathematical Modelling 34 (2010) 3933-3942.

28. C. M. Wang, Y. Y. Zhang, Y. Xiang and J. N. Reddy, Recent studies on buckling of carbon nanotubes, Applied Mechanics Reviews 63 (2010) 030804, 1-18.

29. A. C. Eringen, On differential equations of nonlocal elasticity and solutions of screw dislocation and surface waves, Journal of Applied Physics 54 (1983) 4703-4710.

30. N. Challamel, Lateral-torsional buckling of beams under combined loading - a reappraisal of Papkovitch-Schaefer theorem, International Journal of Structural Stability and Dynamics 7(1) (2007) 55-79.

31. N. Challamel and C. M. Wang, Exact lateral-torsional buckling solutions for cantilevered beams subjected to intermediate and end transverse loads, Thin-Walled Structures 48(1) (2010) $71-76$. 
32. N. Challamel, A. Andrade, D. Camotim and B. M. Milisavlevich, On the flexuraltorsional buckling of cantilever strip beam-columns with linearly varying depth, Journal of Engineering Mechanics 136(6) (2010) 787-800.

33. N. Challamel, On lateral-torsional vibrations of elastic composite beams with interlayer slip, Journal of Sound and Vibration 325 (2009) 1012-1022.

34. N. Challamel, F. Bernard and C. Casandjian, Out-of-plane behaviour of partially composite or sandwich beams by exact and Finite Element Methods, Thin-Walled Structures 48 (2010) 561-580.

35. N. Challamel and C. M. Wang, The small length scale effect for a non-local cantilever beam: A paradox solved, Nanotechnology 19 (2008) 345703.

36. Y. Y. Zhang, C. M. Wang and N. Challamel, Bending, buckling and vibration of hybrid nonlocal beams, Journal of Engineering Mechanics 136(5) (2010) 562-574.

37. N. Challamel, L. Rakotomanana and L. Le Marrec, A dispersive wave equation using nonlocal elasticity, Comptes Rendus Mécanique 337 (2009) 591-595.

38. N. Challamel, A regularization study of some ill-posed gradient plasticity softening beam problems, Journal of Engineering Mathematics 62 (2008) 373-387.

39. N. Challamel, C. Lanos and C. Casandjian, Plastic failure of nonlocal beams, Physical Review E 78 (2008) 026604.

40. N. Challamel, C. Lanos and C. Casandjian, On the propagation of localization in the plasticity collapse of hardening-softening beams, International Journal of Engineering Science 48(5) (2010) 487-506.

41. C. M. Wang, Y. Y. Zhang, S. S. Ramesh and S. Kitipornchai, Buckling analysis of micro and nano-rods/tubes based on nonlocal Timoshenko beam theory, Journal Physics D: Applied Physics 39 (2006) 3904-3909.

42. J. N. Reddy, Nonlocal theories for bending, buckling and vibration of beams, International Journal of Engineering Science 45 (2007) 288-307.

43. H. M. Ma, X. L. Gao and J. N. Reddy, A microstructure-dependent Timoshenko beam model based on a modified couple stress theory, Journal of the Mechanics and Physics of Solids 56 (2008) 3379-3391.

44. Z. P. Bažant, Shear buckling of sandwich, fiber-composite and lattice columns, bearings and helical springs: Paradox resolved, Journal of Applied Mechanics 70 (2003) 75-83.

45. H. Ziegler, Arguments for and against Engesser's buckling formulas, Ingenieur Archiv 52 (1982) 105-113.

46. C. M. Wang, C. Y. Wang and J. N. Reddy, Exact Solutions for Buckling of Structural Members (CRC Series in Computational Mechanics and Applied Analysis, Boca Raton, Florida, 2005).

47. N. Challamel and C. M. Wang, On lateral-torsional buckling of non-local beams, Advances in Applied Mathematics and Mechanics 3 (2010) 389-398.

48. A. Tounsi, Improved theoretical solution for interfacial stresses in concrete beams strengthened with FRP plate, International Journal of Solids and Structures 43 (2006) $4154-4174$. 